\title{
Food and Nutrient Intake over the Course of the Menstrual Cycle - Cycles Iceland
}

\author{
Bryndis Eva Birgisdóttir $^{1}$, Geir Gunnlaugsson ${ }^{2}$ and Virginia Vitzhum ${ }^{3}$ \\ ${ }^{1}$ Unit for Nutrition Research, Faculty of Food Science and Nutrition and the Landspitali University Hospital, \\ Reykjavík, Iceland, \\ ${ }^{2}$ Faculty of Sociology, Anthropology and Folkloristics, University of Iceland, Reykavík, Iceland and \\ ${ }^{3}$ Department of Anthropology, Indiana University, Bloomington, Indiana, USA
}

\section{Abstract}

Introduction: In Iceland there are extreme seasonal differences in natural light. How these might affect the physiology of young women is largely unknown and prompted The Cycles-Iceland study. Both seasonal differences and the menstrual cycle have been suggested to affect womeńs food consumption and therefore nutrient intake. The aim of this part of the Cycles-Iceland study was to investigate food consumption and nutrient intake at different time points of the year and over the menstrual cycle.

Materials and methods: In total 23 young women, mean (SD) age 31.6 (5.5) years, gave six 24-h dietary recalls each. These were given at the start, middle and at the end of one menstrual cycle, in both summer and winter seasons. The women were asked about various background factors while other lifestyle habits were also measured such as physical activity and sleep and light exposure. The women also gave biological samples, blood spots, urine and saliva for evaluation of hormonal and immune biomarkers.

Results: The group scored above average on a healthy diet evaluation, based on the Icelandic Food Based Dietary Guidelines. First crude results indicate difference in choice of certain food items and therefore nutrient intake ex. sugar $(p<0.001)$ and fiber intake $(p<$ $0.010)$, depending on the position in menstrual cycle. Difference in food choices were also seen between winter and summer time $(\mathrm{p}<$ $0.04)$.

Discussion: Food and nutrient intake seems to be affected by the position of women in their menstrual cycle. Whether this might be related to hormonal status, inflammatory factors and other lifestyle factors needs further investigation. The results from Cycles-Iceland will contribute to better understanding of metabolic and health effects of natural seasonal cycles in the northern hemisphere.

Funding: US National Science Foundation (NSF1319663), U.S./Iceland Fulbright Program, and Indiana University

\section{Conflict of Interest}

There is no conflict of interest 